\title{
Si può fomentare anche il bene: è soltanto più raro
}

\author{
Kevin De Vecchis
}

PUBBLICATO: 22 MAGGIO 2020

\section{Quesito:}

Diversi lettori chiedono spiegazioni riguardo alla semantica odierna e all'evoluzione passata di fomentare. In particolare, le domande vertono sulla possibile accezione positiva del verbo. Gli esempi riportati dagli utenti sono due: "sono stati erogati dei distributori d'acqua negli spazi comuni. Diffondete la voce per fomentarne l'utilizzo a tutti i colleghi", dove il verbo con valore transitivo assume il significato di 'incoraggiare, promuovere un'azione auspicabile'; "sono fomentato perché mi accadrà questa cosa”, dove il participio passato segue il verbo essere e l'accezione è spostata sull'area semantica di 'essere agitati o in fibrillazione per un evento prossimo', a prescindere dal fatto che questo sia vantaggioso o svantaggioso per il soggetto dell'enunciato.

\section{Si può fomentare anche il bene: è soltanto più raro}

Il verbo fomentare è transitivo ed è una voce di derivazione dotta dal lat. tardo fomentāre, derivante dal lat. foméntu $(m)$ e a sua volta da fovēre 'riscaldare' (cfr. DELI).

Il primo significato che viene riportato dalla lessicografia italiana è quello di 'applicare un fomento, un impacco per lo più caldo, a una parte del corpo (per calmare un dolore, per curare una contusione, una ferita); curare con fomenti' (cfr. GDLI); tuttavia, tale significato è ormai etichettato con ant. 'antico, fuori dall'uso' dal GDLI e con [BU] 'di basso uso' dal GRADIT. Una seconda accezione, traslata e affine al precedente, è quella di 'infondere calore vivificante; far maturare, curare, nutrire; invigorire; tenere caldo, riscaldare' (cfr. GDLI, GRADIT), ma anch'essa di scarsa diffusione.

Al giorno d'oggi il verbo è adoperato soprattutto nel suo uso figurato di "incitare, istigare: fomentare l'odio, la discordia, la passione | (est.) promuovere, suscitare: fomentare una rivolta" (cfr. Zingarelli 20rg), come confermano anche il GRADIT ("[CO] fig., istigare, attizzare: f. una rivolta, f. disordini, f. l'odio, la ribellione") e il Sabatini-Coletti ("peggiorare una situazione già compromessa, istigando con malignità le persone coinvolte").

L'uso odierno suggerisce, dunque, un impiego di fomentare con valore negativo. Cosi come era anche in passato. Il GDLI riporta come significati: 'suscitare, promuovere, istigare (una guerra, una sedizione, ecc.); incitare, inasprire (un dissidio, una ribellione); stimolare (un sospetto, una calunnia)'; e ancora 'sobillare, istigare qualcuno a commettere un'azione disonesta o malvagia' o 'tener vivo, avvalorare, lusingare, secondare; accendere, attizzare, risvegliare; eccitare, inasprire, esasperare (un sentimento per lo più violento, un'inclinazione per lo più malvagia, ecc.)'. Qui alcuni esempi:

L'ufficio [di un pontefice romano]... era proibire, non fomentare, le violenze e le guerre tra i principi cristiani (F. Guicciardini, Legazione di Spagna)

Intimarono al console che guardasse bene di non far deposizione al podestà dell'accaduto, ...di non ciarlare, di non fomentar le ciarle de' villani, per quanto aveva cara la speranza di morir di malattia. (A. Manzoni, I promessi sposi, VIII) 
La risposta di Stratocle starebbe molto bene in bocca de' poeti, de' musici, degli antichi filosofi, della natura, delle illusioni medesime, di tutti quelli che sono accusati d'avere introdotti o fomentati, d'introdurre o fomentare o promuovere de' begli errori nel genere umano, o in qualche nazione o in qualche individuo. (G. Leopardi, Detti memorabili di Filippo Ottonieri, Operette morali)

Tuttavia, il verbo fomentare può assumere anche accezioni positive. Il GDLI riporta pure i significati di 'spronare, incitare, stimolare, promuovere (l'ingegno, il valore, la virtù, ecc.)', 'stimolare, stuzzicare (la curiosità, l'appetito)', 'eccitare (il riso)', 'sostenere, appoggiare, favorire (una persona, un'opinione, un partito, un piano d'azione, uno stato di cose); incoraggiare (un'intenzione, una speranza, un'impresa, una credenza)', cosi come si legge da alcuni esempi:

Fu de' più antichi abitatori dell'Italia l'uso de' gladiatori, ...parendo forse loro che potesse molto contribuire a fomentar valore e bravura. (S. Maffei, Verona illustrata, V, p. II)

La compiacenza di questi miseri applausi, ...non potea più fomentare gl'ingegni ad aspirare con più veglie e sudori ad una gloria più estesa. (U. Foscolo, Articoli di critica e di polemica)

Nella stessa maniera dico io delle antiche istituzioni... tendenti a fomentare l'entusiasmo, le illusioni, il coraggio, l'attività, il movimento, la vita. (G. Leopardi, Zibaldone di pensieri)

Una contenutezza cortese c'era per riguardo al commissario ed alla sua signora, mentre giusto il commissario cercava di fomentare intorno un senso di famigliarità e d'allegria. (G. Deledda, Il paese del vento, p. 198)

Al giorno d'oggi, tale valore semanticamente positivo è segnalato dallo Zingarelli 2019, che scrive 'favorire, incoraggiare', etichettandolo però come lett(erario). Effettivamente, una ricerca in sincronia nel corpus la Repubblica dimostra come fomentare sia prevalentemente affiancato da complementi oggetti che costituiscono sentimenti o azioni negative. Il verbo (nella forma dell'infinito) negli articoli

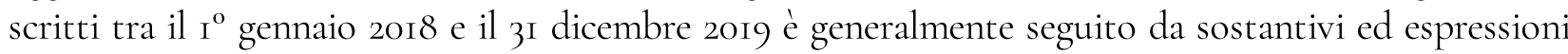
quali: "la protesta", "la discordia", "gli episodi di razzismo", "la violenza fisica e verbale", "il clima [di razzismol", "l'odio", "la rabbia", "la rivolta", "la guerra", ecc. Se intendiamo parole come "la rivolta" e "la protesta" come semanticamente negative, allora gli unici riscontri della sequenza V + Ogg. con valore positivo sono soltanto 5 su un totale di I42 attestazioni:

«Continuerò a fomentare quest'amore, a credere che Palermo può e merita di cambiare» [20.05.2018]

«ll testo rientrava fra i documenti ufficiali della diocesi cilena. Era intitolato "Orientaciones que fomentan el buen trato y la sana convivencia pastoral" (Orientamenti per fomentare il buon rapporto e la sana convivenza pastorale) e nelle scorse ore aveva provocato non poche critiche tra i fedeli sempre più scottati dalle coperture concesse ad alcuni preti pedofili» [02.10.2018]

«Lunedi tu prendi e te ne vai in prefettura - incalza Daniela Giaconi, considerata dagli inquirenti la vera tessitrice della complessa rete di onlus per il drenaggio di denaro pubblico, unica finita in carcere - e cerchi di fomentare il pagamento di questa fattura!» [03.07.2019]

«Proprio contro le politiche protezionistiche in ambito economico l'attacco è netto: per Tria "danneggiano i Paesi coinvolti", che invece dovrebbero fomentare la "collaborazione" tra di loro" [10.07.2019]

«Fomentare il senso di appartenenza alla squadra e lo spirito di competizione sembra il suo motto». [20.II.2019] 
La situazione è ben diversa se consideriamo il possibile uso riflessivo del verbo, di cui pare non esservi traccia nella lessicografia italiana, oppure la presenza del participio passato fomentato dopo una forma del verbo essere, documentata da esempi recenti in rete. In questi casi il significato è quello, segnalato dai nostri lettori, di 'essere eccitati' e le accezioni assumono un carattere prevalentemente positivo. Si può essere, infatti, fomentati con o per qualcuno o qualcosa (un personaggio televisivo, un cantante, un concerto, un film, un evento, ecc.) o fomentati in senso assoluto. Qui di seguito alcuni esempi tratti dalla rete:

Stasera mi sono fomentato con quel concerto... quante emozioni! [Twitter 2013]

Mi sono emozionato, mi sono fomentato, mi sono divertito, mi sono commosso. Grazie Vasco, grazie di esistere [Twitter, 2016]

Quanto sono fomentato per scoprire la Capitale della Cultura 20I8! [Twitter, 20I7]

Comunque oggi sono fomentato con lo studio, si procede alla grande [Twitter, 20I9]

Sono fomentato per la prossima stagione, vinciamo tutto. C'è stato un cambio di marcia in società, lo percepisco [Twitter, 2019]

Inoltre, in rete è possibile riscontrare l'uso anche del sostantivo fomento con il significato di 'eccitazione', nell'espressione che fomento!, che risulta impiegata soprattutto in ambito calcistico e da utenti provenienti da Roma, luogo in cui nel 1994 nasce anche un famoso gruppo musicale chiamato Colle der fomento.

Oggi sono andata a vedere l'arrivo di Dzeko... Non immaginate che fomento. Te amo Edin [Twitter, $2015]$

Che fomento questo sole, ho fatto 3 lavatrici, sono in pieno hype, fra poco lavo e stendo pure i piatti e le pentole [Twitter, 2019]

In conclusione, oggi il verbo transitivo fomentare è maggiormente usato come sinonimo di istigare ed esprime, dunque, un incitamento a qualcosa di riprovevole. Il suo utilizzo nel senso di 'incoraggiare o favorire azioni o sentimenti positivi' è invece decisamente più raro, ma non può considerarsi scorretto. Certo da questa eccezione si è sviluppato l'uso riflessivo di fomentarsi, la cui semantica si accosta piuttosto a quella di verbi come eccitarsi, gasarsi ed esaltarsi, e ha permesso uno slittamento da complementi oggetti inanimati (fomentare qualcosa) ad animati (fomentare qualcuno); da qui probabilmente è nato l'uso di fomentato come aggettivo nel senso di 'eccitato', usato per lo più come predicato nominale ( $m i$ sono fomentato $>$ sono fomentato), che potrebbe in futuro portare a un uso intransitivo del verbo fomentare con lo stesso significato del riflessivo.

Un esempio simile è costituito dalla coppia di verbi transitivi gasare/gassare: il complemento oggetto retto da entrambi è ad oggi anche animato e non più soltanto inanimato ('rendere effervescente un liquido'); inoltre il primo verbo è utilizzato in un'accezione positiva ('eccitare') e conosce anche la forma riflessiva gasarsi; il secondo, invece, ha una semantica negativa 'sottoporre all'azione di gas tossici' (Zingarelli 2019).

Sebbene non risulti registrato nei dizionari italiani, l'uso riflessivo di fomentare appare molto esteso, soprattutto a Roma, che potrebbe essere un polo di irradiazione, dove sono attestati sia fomentà nel senso di 'eccitare, aizzare', sia fomentàsse nei significati di 'eccitarsi, agitarsi eccessivamente' e 'montarsi 
la testa', sia l'aggettivo fomentato 'eccitato', tutti e tre giovanilismi, cosi come registra il Vocabolario del romanesco contemporaneo di Paolo D'Achille e Claudio Giovanardi (la cui lettera F è attualmente in stampa).

\section{Cita come:}

Kevin De Vecchis, Si può fomentare anche il bene: è soltanto più raro, "Italiano digitale", 2020, XIII, 2020/2 (aprile-giugno)

DOI: $10.35948 / 2532-9006 / 2020.3333$

Copyright 2020 Accademia della Crusca

Pubblicato con licenza creative commons CC BY-NC-ND 